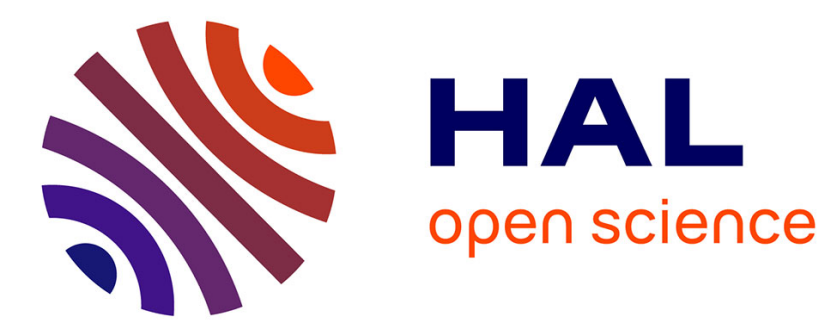

\title{
Characterization of microsatellite markers in the interspecific hybrid Phytophthora alni ssp. alni, and cross-amplification with related taxa
}

\author{
Renaud Ioos, Benoît Barres, Axelle Andrieux, Pascal Frey
}

\section{To cite this version:}

Renaud Ioos, Benoît Barres, Axelle Andrieux, Pascal Frey. Characterization of microsatellite markers in the interspecific hybrid Phytophthora alni ssp. alni, and cross-amplification with related taxa. Molecular Ecology Notes, 2007, 7 (1), pp.133-137. 10.1111/j.1471-8286.2006.01554.x . hal-01136981

\author{
HAL Id: hal-01136981 \\ https://hal.science/hal-01136981
}

Submitted on 30 Mar 2015

HAL is a multi-disciplinary open access archive for the deposit and dissemination of scientific research documents, whether they are published or not. The documents may come from teaching and research institutions in France or abroad, or from public or private research centers.
L'archive ouverte pluridisciplinaire HAL, est destinée au dépôt et à la diffusion de documents scientifiques de niveau recherche, publiés ou non, émanant des établissements d'enseignement et de recherche français ou étrangers, des laboratoires publics ou privés. 
PRIMER NOTE

\title{
Characterization of microsatellite markers in the interspecific hybrid Phytophthora alni ssp. alni, and cross-amplification with related taxa
}

\author{
RENAUD IOOS, ${ }^{*}+$ BENOÎT BARRÈS, ${ }^{*}$ AXELLE ANDRIEUX* and PASCAL FREY* \\ *INRA, Equipe de Pathologie Forestière, UMR 1136 Interactions Arbres - Microorganismes, IFR 110, 54280 Champenoux, France, \\ +Laboratoire National de la Protection des Végétaux, UMAF, Domaine de Pixérécourt, 54220 Malzéville, France
}

\begin{abstract}
Phytophthora alni ssp. alni is an interspecific hybrid oomycete causing a large-scale decay of alders throughout Europe. In this study we developed a set of $\mathbf{1 0}$ microsatellite markers that shows promise for population studies and for studying hybridization events between the parental species of the hybrid. Moreover, the genotype and the ploidy of the different subspecies of $P$. alni might be inferred from the quantitative ratio of amplified genomespecific alleles. Nine primer pairs cross amplified with the related species Phytophthora cambivora and Phytophthora fragariae and yielded distinct alleles.
\end{abstract}

Keywords: genotyping, oomycete, polyploidy

Received 29 June 2006; revision 7 August 2006

Phytophthora alni ssp. alni (Paa) is a recently described interspecific hybrid oomycete causing a lethal disease of alder throughout Europe (Brasier et al. 2004). Two other taxa close to $P a a$ are isolated from diseased alders or from soil beneath alders: Phytophthora alni ssp. uniformis (Pau) and Phytophthora alni ssp. multiformis (Pam). All three taxa are phylogenetically very close to Phytophthora cambivora $\left(P_{C}\right)$ and Phytophthora fragariae (Pf) (Brasier et al. 1999). Previously hypothesized to originate from genetic breakdown of $\mathrm{Paa}$ (Brasier et al. 1999), Pam and Pau were actually suggested to represent distinct species and to have generated $P a a$ through interspecific hybridization (Ioos et al. 2006). In addition, Brasier et al. (1999) reported that Paa was a near tetraploid species $(4 n+$ 2 ) in respect with the typical diploid (2n) status of the Phytophthora genus, and that Pam and Pau were aneuploid species with $2 n+4$ to $2 n+7$, and $2 n+2$ chromosomes, respectively. However, Ioos et al. (2006) demonstrated that three divergent alleles for four unlinked single copy nuclear genes were observed within the Paa genome. Two of them matched the two alleles also found in Pam and the third one corresponded to the single allele found in Pau. As these results contrasted with the ploidy levels reported, and as little is known about the genetic polymorphism within Paa

Correspondence: P. Frey, Fax: +33 383394069,

E-mail: frey@nancy.inra.fr and the two other taxa, we isolated and characterized microsatellite loci in Paa. These codominant markers are especially useful to address questions relating to origin and genetic diversity of fungi (Weising et al. 1995).

Microsatellite markers were isolated from Paa using enrichment libraries. DNA was extracted from $P a a$ isolate PAA130 using DNeasy Plant Mini Kit (QIAGEN). Approximately $500 \mathrm{ng} \mu \mathrm{L}^{-1}$ DNA were recovered and used for the construction of five enrichment libraries using biotinylated oligoprobes $\left[(\mathrm{AC})_{13^{\prime}}(\mathrm{AG})_{13^{\prime}}(\mathrm{ACG})_{6},(\mathrm{AAC})_{6}\right.$ and $\left.(\mathrm{AAG})_{6}\right]$ and streptavidin-coated magnetic beads, according to Dutech et al. (2000). The libraries were cloned using a TOPO TA Cloning kit (Invitrogen). For each of the five enrichments, 288 clones were screened by colony blot following a protocol developed by Estoup et al. (1993). A total of 172 positive clones were subsequently size-screened by polymerase chain reaction (PCR) using vector's primers and 43 clones with insert between 400 and $1000 \mathrm{bp}$ were retained for sequencing. Eighteen primer pairs could be designed from the sequences, either manually or using PRIMER 3 software (Rozen \& Skaletski 2000). The primer pairs were tested by PCR with a panel of 6 Paa 4 Pam, 8 Pau, 2 Pc and 2 Pf isolates, from various geographical origins in Europe and displaying different mitotypes according to Ioos et al. (2006) (Table 1). PCR conditions were optimized for each primer pair. PCR was conducted in a $20-\mu \mathrm{L}$ volume containing $15-20 \mathrm{ng}$ of 
134 PRIMER NOTE

Table 1 List of the isolates used in the study. Mitotypes of the isolates determined as described in Ioos et al. (2006)

\begin{tabular}{|c|c|c|c|c|c|}
\hline Species & Isolate & Mitotype & Host & $\begin{array}{l}\text { Geographical } \\
\text { origin }\end{array}$ & $\begin{array}{l}\text { Year } \\
\text { isolated }\end{array}$ \\
\hline \multirow[t]{34}{*}{ Phytophthora alni ssp. alni } & PAA129 & $\mathrm{U}$ & Alnus glutinosa & SW France & 2003 \\
\hline & PAA130* & M & Alnus glutinosa & NE France & 2003 \\
\hline & PAA143 & $\mathrm{M}^{\prime \prime}$ & Alnus glutinosa & Poland & 2002 \\
\hline & PAA151 & $\mathrm{U}$ & Alnus glutinosa & NE France & 2004 \\
\hline & PAA162 & $\mathrm{U}$ & Alnus glutinosa & Germany & 2004 \\
\hline & PAAa2' & $\mathrm{U}$ & Alnus glutinosa & NE France & 2005 \\
\hline & PAAe3 & M & Alnus glutinosa & NE France & 2005 \\
\hline & PAA151 & $\mathrm{U}$ & Alnus glutinosa & NE France & 2004 \\
\hline & PAA70 & M & Alnus glutinosa & the Netherlands & Unknown \\
\hline & PAAa2 & M & Alnus glutinosa & NE France & 2005 \\
\hline & PAA38 & M & Alnus glutinosa & N France & 2002 \\
\hline & PAA74 & $\mathrm{U}$ & Alnus glutinosa & Scotland & 2000 \\
\hline & PAA75 & M & Alnus viridis & Scotland & 2000 \\
\hline & PAA81 & $\mathrm{U}$ & Alnus glutinosa & England & 1997 \\
\hline & PAA82 & M & Alnus glutinosa & England & 1996 \\
\hline & PAAe1 & M & Alnus glutinosa & NE France & 2005 \\
\hline & PAA85 & M & Alnus glutinosa & England & Unknown \\
\hline & PAA86 & M & Alnus glutinosa & Belgium & 1999 \\
\hline & PAA88 & M & Alnus glutinosa & Belgium & 2001 \\
\hline & PAA91 & $\mathrm{M}^{\prime}$ & Alnus glutinosa & Hungary & 2001 \\
\hline & PAA112 & $\mathrm{M}$ & Alnus glutinosa & NW France & 2003 \\
\hline & PAA114 & M & Alnus glutinosa & NE France & 2002 \\
\hline & PAA134 & M & Alnus glutinosa & Germany & 2000 \\
\hline & PAA141 & $\mathrm{U}^{\prime}$ & Alnus glutinosa & Austria & Unknown \\
\hline & PAA144 & $\mathrm{M}^{\prime \prime}$ & Alnus glutinosa & Poland & 2003 \\
\hline & PAA178 & $\mathrm{M}^{\prime \prime}$ & Alnus glutinosa & Poland & 2003 \\
\hline & PAA185 & $\mathrm{M}$ & Alnus glutinosa & NE France & 2004 \\
\hline & PAA194 & M & Alnus glutinosa & NW France & 2005 \\
\hline & PAAd6' & $\mathrm{U}$ & Alnus glutinosa & NW France & 2005 \\
\hline & PAAa10 & M & Alnus glutinosa & N France & 2005 \\
\hline & PAAc10 & M & Alnus glutinosa & N France & 2005 \\
\hline & PAAd2' & $M^{\prime}$ & Alnus glutinosa & NE France & 2005 \\
\hline & PAAe2' & $\mathrm{M}$ & Alnus glutinosa & NE France & 2005 \\
\hline & PAAa11' & M & Alnus glutinosa & Germany & 2005 \\
\hline \multirow[t]{5}{*}{ Phytophthora alni ssp. multiformis } & PAM54 & M & Alnus glutinosa & NW France & 2000 \\
\hline & PAM71 & M & Alnus glutinosa soil & the Netherlands & 1995 \\
\hline & PAM90 & M & Alnus glutinosa soil & the Netherlands & 1995 \\
\hline & PAM186 & $M^{\prime}$ & Alnus glutinosa & Belgium & 2001 \\
\hline & PAM73 & nd & Alnus glutinosa & England & 1996 \\
\hline \multirow[t]{13}{*}{ Phytophthora alni ssp. uniformis } & PAU60 & $\mathrm{U}^{\prime \prime}$ & Alnus glutinosa & NE France & 1999 \\
\hline & PAU84 & $\mathrm{U}^{\prime}$ & Alnus glutinosa & Sweden & 1999 \\
\hline & PAU87 & $\mathrm{U}$ & Alnus glutinosa & Belgium & 2001 \\
\hline & PAU89 & $\mathrm{U}$ & Alnus cordata & Italy & 2000 \\
\hline & PAU142 & $\mathrm{U}$ & Alnus glutinosa & Slovenia & 2000 \\
\hline & PAU188 & $\mathrm{U}$ & Alnus incana & Belgium & 2001 \\
\hline & PAUb3 & $\mathrm{U}^{\prime \prime}$ & Alnus glutinosa & NE France & 2005 \\
\hline & PAUc6' & $\mathrm{U}$ & Alnus glutinosa & NE France & 2005 \\
\hline & PAU187 & $\mathrm{U}$ & Alnus glutinosa & Belgium & 2001 \\
\hline & PAU96 & $\mathrm{U}$ & Alnus glutinosa & Hungary & 1999 \\
\hline & PAU97 & $\mathrm{U}$ & Alnus glutinosa soil & Hungary & 1999 \\
\hline & PAU98 & $\mathrm{U}$ & Alnus glutinosa soil & Hungary & 1999 \\
\hline & PAUb3 & $\mathrm{U}^{\prime}$ & Alnus glutinosa & NE France & 2005 \\
\hline \multirow{4}{*}{ Phytophthora cambivora } & PCJC17 & $\mathrm{C} 2$ & Quercus sp. soil & NE France & 1999 \\
\hline & PC643 & $\mathrm{C} 1$ & Castanea sativa soil & SW France & 2000 \\
\hline & PC463 & $\mathrm{C} 2$ & Castanea sativa & SW France & 1994 \\
\hline & PCga1 & $\mathrm{C} 2$ & Quercus sp. soil & NE France & 1999 \\
\hline
\end{tabular}


Table 1 Continued

\begin{tabular}{|c|c|c|c|c|c|}
\hline Species & Isolate & Mitotype & Host & $\begin{array}{l}\text { Geographical } \\
\text { origin }\end{array}$ & $\begin{array}{l}\text { Year } \\
\text { isolated }\end{array}$ \\
\hline & PC99428 & $\mathrm{C} 2$ & Castanea sativa & France & 1999 \\
\hline & PCst3r1 & $\mathrm{C} 2$ & Quercus petraea & SW France & 1999 \\
\hline & PC627 & $\mathrm{C} 2$ & Castanea sativa & Italy & 1999 \\
\hline & PC1a21 & $\mathrm{C} 2$ & Quercus sp. soil & NW France & 1999 \\
\hline & PC4n444 & $\mathrm{C} 2$ & Castanea sativa & NW France & 2004 \\
\hline & PC4n1125 & $\mathrm{C} 2$ & Castanea sativa & NW France & 2004 \\
\hline & PC051422 & $\mathrm{C} 2$ & Castanea sativa & NW France & 2005 \\
\hline \multirow[t]{3}{*}{ Phytophthora fragariae var. fragariae } & PFF309 & FF & Fragaria $\times$ ananassa & Great Britain & 1962 \\
\hline & PFFCSL & FF & Fragaria $\times$ ananassa & Unknown & Unknown \\
\hline & PFF209 & FF & Fragaria $\times$ ananassa & England & 1946 \\
\hline \multirow[t]{5}{*}{ Phytophthora fragariae var. rubi } & PFR109 & FR & Rubus sp. & Great Britain & 1991 \\
\hline & PFR163 & FR & Rubus sp. & France & Unknown \\
\hline & PFR59 & FR & Rubus sp. & Scotland & Unknown \\
\hline & PFRCSL & FR & Rubus sp. & England & Unknown \\
\hline & PFR96795 & FR & Rubus sp. & Scotland & 1985 \\
\hline Phytophthora cactorum & CAC4810 & nd & Unknown & France & Unknown \\
\hline Phytophthora cinnamomi & DSF970060 & nd & Quercus suber & France & 1999 \\
\hline Phytophthora citricola & AUL045 & nd & Alnus glutinosa & France & 1999 \\
\hline Phytophthora citrophthora & 2 N1021 & nd & Rosa sp. & France & 2002 \\
\hline Phytophthora cryptogea & 990675 & nd & Actinidia sinensis & France & 1999 \\
\hline Phytophthora erythroseptica & 960713 & nd & Polygonum oberti & France & 1999 \\
\hline Phytophthora europaea & AL5 & nd & Quercus sp. soil & France & 1998 \\
\hline Phytophthora gonapodyides & Gonap4 & nd & Quercus sp. soil & France & 1998 \\
\hline Phytophthora humicola & $3 \mathrm{~N} 1245 \mathrm{j}$ & nd & Alnus glutinosa soil & France & 2003 \\
\hline Phytophthora inundata & 9500802 & nd & Alnus glutinosa soil & France & 1998 \\
\hline Phytophthora lateralis & 98093-1 PV & nd & Chamaecyparis sp. & France & 1998 \\
\hline Phytophthora megasperma & BK1 & nd & Quercus sp. soil & France & 1998 \\
\hline Phytophthora nicotianae & 960579 & nd & Nicotiana tabacum & France & 1996 \\
\hline Phytophthora taxon forest soil & 8CARPPOC1 & nd & Quercus sp. soil & France & 1998 \\
\hline Phytophthora palmivora & 970423 & nd & Hedera sp. & France & 1997 \\
\hline Phytophthora parasitica & 970029 & nd & Lycopersicon sp. & France & 1997 \\
\hline Phytophthora taxon Pgchlamydo & Haye,3,1 & nd & Quercus sp. soil & France & 1998 \\
\hline Phytophthora pseudosyringae & EW5 & nd & Quercus sp. soil & France & 1998 \\
\hline Phytophthora psychrophila & FF20 & nd & Quercus sp. soil & France & 1998 \\
\hline Phytophthora quercina & FNA & nd & Quercus sp. soil & France & 1999 \\
\hline Phytophthora ramorum & 2 N083 & nd & Rhododendron sp. & France & 2002 \\
\hline Phytophthora sojae & 443 & nd & Glycine $\max$ & Unknown & Unknown \\
\hline Phytophthora syringae & $2 \mathrm{JZ2}$ & nd & Quercus sp. soil & France & 1999 \\
\hline
\end{tabular}

*, isolate used for microsatellite isolation; nd, not determined.

template DNA, $2 \mu \mathrm{L}$ of $10 \times$ polymerase buffer, $1.5 \mathrm{~mm}$ $\mathrm{MgCl}_{2}, 0.2 \mathrm{~mm} \mathrm{dNTP}, 0.2 \mu \mathrm{M}$ of each primer, $0.5 \mathrm{U}$ of Taq polymerase (Sigma) and water was added to $20 \mu \mathrm{L}$. Touchdown PCR conditions were first five cycles at $94{ }^{\circ} \mathrm{C}$ for $30 \mathrm{~s}, 66-62{ }^{\circ} \mathrm{C}$ (see initial annealing temperatures, Table 2) for $30 \mathrm{~s}$ and $72{ }^{\circ} \mathrm{C}$ for $1 \mathrm{~min}$; followed by 8 cycles at $94{ }^{\circ} \mathrm{C}$ for $30 \mathrm{~s}, 66-55{ }^{\circ} \mathrm{C}$ minus $0.5^{\circ} \mathrm{C}$ per cycle for $30 \mathrm{~s}$, and $72{ }^{\circ} \mathrm{C}$ for $1 \mathrm{~min} ; 22$ cycles at $94^{\circ} \mathrm{C}$ for $30 \mathrm{~s}, 62-51^{\circ} \mathrm{C}$ for $30 \mathrm{~s}$, and $72{ }^{\circ} \mathrm{C}$ for $1 \mathrm{~min}$; and a final elongation step at $65^{\circ} \mathrm{C}$ for $30 \mathrm{~min}$. All retained forward primers were labelled to allow size and dye multiplexing. Allele sizes were determined on a CEQ 8000 Genetic Analysis System (Beckman Coulter). Eight out of the 18 primer pairs tested yielded no polymorphism or unclear pattern and were therefore discarded. The remaining 10 primer pairs were tested by PCR on a larger collection of Paa, Pau, Pam, P. cambivora and P. fragariae isolates as well as with 23 other Phytophthora spp. (Table 1).

Except PA12-F/R that were specific to Paa and Pam but gave no size polymorphism, all the primers retained yielded an amplicon with Paa, Pau, Pam, Pc and Pf, confirming their close kinship. In $P a a$, the number of alleles for the nine polymorphic loci ranged from two to five indicating a low level of allelic variation, consistent with this hybrid species being of recent origin (Brasier et al. 2004; Ioos et al. 2006). In addition, all the alleles observed in Paa were those encountered either in Pam or in Pau (Table 2), strengthening the 
Table 2 Primer sequences and characteristics for the nine polymorphic microsatellite loci isolated from Phytophthora alni ssp. alni. All reverse PCR primers were fluorescently labelled

\begin{tabular}{|c|c|c|c|c|c|c|c|c|c|c|c|c|}
\hline \multirow[b]{2}{*}{ Locus } & \multirow[b]{2}{*}{$\begin{array}{l}\text { Repeat } \\
\text { motif }\end{array}$} & \multirow[b]{2}{*}{$\begin{array}{l}\text { GenBank } \\
\text { Accession no. }\end{array}$} & \multirow[b]{2}{*}{ Primer sequence $\left(5^{\prime}-3^{\prime}\right)$} & \multirow[b]{2}{*}{$\begin{array}{l}\text { Size } \\
\text { (bp) }\end{array}$} & \multirow[b]{2}{*}{$\begin{array}{l}\text { Annealing } \\
\mathrm{T}^{\circ} \mathrm{C}^{*}\end{array}$} & \multicolumn{7}{|c|}{ Total number of different alleles/no. of alleles per individual (allele sizes) } \\
\hline & & & & & & P. alni ssp. alni & $\begin{array}{l}\text { P. alni ssp. } \\
\text { multiformis }\end{array}$ & $\begin{array}{l}\text { P. alni ssp. } \\
\text { uniformis }\end{array}$ & P. cambivora & $\begin{array}{l}\text { P. fragariae } \\
\text { var fragariae }\end{array}$ & $\begin{array}{l}\text { P. fragariae } \\
\text { var rubi }\end{array}$ & $\begin{array}{l}\text { other } \\
\text { species }\end{array}$ \\
\hline PA3 & $\begin{array}{l}(\mathrm{CAA}) \mathrm{GA} \\
(\mathrm{CAA})_{5}\end{array}$ & DQ665899 & $\begin{array}{l}\text { F: CTTGGATAGAGCCGTCGTTC } \\
\text { R: TCCTACTGTTGGGAGCAAGG }\end{array}$ & 201 & $62{ }^{\circ} \mathrm{C}$ & $\begin{array}{l}3 / 2+(198 \\
212,216)\end{array}$ & $\begin{array}{l}2 / 1-2+ \\
(198,216)\end{array}$ & $\begin{array}{l}2 / 1-2 \\
(198,212)\end{array}$ & $\begin{array}{l}4 / 3(210,211, \\
213,215)\end{array}$ & $1 / 1(217)$ & $1 / 1(217)$ & - \\
\hline PA6 & $\begin{array}{l}(\mathrm{CAA})_{11} \\
\operatorname{TAA}(\mathrm{CAA})\end{array}$ & DQ665900 & $\begin{array}{l}\text { F: AACACCGCGTTGAAGACG } \\
\text { R: GTAGCCACCGCACATGAATC }\end{array}$ & 303 & $66^{\circ} \mathrm{C}$ & $\begin{array}{l}3 / 3+(278 \\
284,287)\end{array}$ & $1 / 1(278)$ & $\begin{array}{l}3 / 3(278 \\
284,287)\end{array}$ & $2 / 2(278,281)$ & $\begin{array}{l}2 / 1-2(275 \\
288)\end{array}$ & $\begin{array}{l}2 / 2 \\
(275,288)\end{array}$ & - \\
\hline PA8 & $\begin{array}{l}(\mathrm{ACA})_{5} \\
\mathrm{~T}(\mathrm{ACA})\end{array}$ & DQ665901 & $\begin{array}{l}\text { F: GGTCAGCCAAGAGCAAAGAG } \\
\text { R: CTGTGAGCTGCAAGAAGCAG }\end{array}$ & 376 & $66^{\circ} \mathrm{C}$ & $\begin{array}{l}5 / 3-5+(356 \\
359,361 \\
367,368)\end{array}$ & $\begin{array}{l}3 / 2-3+(356 \\
359,368)\end{array}$ & $\begin{array}{l}2 / 2 \\
(361,367)\end{array}$ & $\begin{array}{l}2 / 1-2(367, \\
369)\end{array}$ & $\begin{array}{l}2 / 2(364 \\
367)\end{array}$ & $1 / 1(367)$ & $\begin{array}{l}\text { P.europaed } \\
(368)\end{array}$ \\
\hline PA11 & $\begin{array}{l}(\mathrm{CAA})_{5} \mathrm{CAG} \\
(\mathrm{CAA})\end{array}$ & DQ665902 & $\begin{array}{l}\text { F: TGCAAACAGTGCGTCTCTTC } \\
\text { R: CCTAGATCCAGCGACAGCTC }\end{array}$ & 226 & $62^{\circ} \mathrm{C}$ & $\begin{array}{l}2 / 2+ \\
(226,232)\end{array}$ & $\begin{array}{l}2 / 1-2+ \\
(226,232)\end{array}$ & $1 / 1(226)$ & $\begin{array}{l}2 / 1-2(224, \\
225)\end{array}$ & $1 / 1(226)$ & $1 / 1(225)$ & - \\
\hline PA14 & $\begin{array}{l}(\mathrm{CT})_{7}(\ldots) \\
(\mathrm{TTTC})_{4}\end{array}$ & DQ665904 & $\begin{array}{l}\text { F: TGGCAAACAGACACGAAGTC } \\
\text { R: GAAACCCAGTCATCCGAGAG }\end{array}$ & 173 & $62{ }^{\circ} \mathrm{C}$ & $\begin{array}{l}3 / 3+(173 \\
175,177)\end{array}$ & $\begin{array}{l}2 / 2+(173 \\
175)\end{array}$ & $1 / 1(177)$ & $\begin{array}{l}4 / 2(174,177 \\
179,181)\end{array}$ & $\begin{array}{l}2 / 2(168 \\
174)\end{array}$ & $\begin{array}{l}2 / 2 \\
(175,177)\end{array}$ & - \\
\hline PA17 & $\begin{array}{l}(\mathrm{GTC})_{4}(\ldots) \\
(\mathrm{GC})_{4}\end{array}$ & DQ665905 & $\begin{array}{l}\text { F: AGCGACAATGCAGGAAGC } \\
\text { R: CTGTCTGGGCATTCATGTCG }\end{array}$ & 317 & $62^{\circ} \mathrm{C}$ & $2 / 2(313,317)$ & $\begin{array}{l}2 / 2(313 \\
317)\end{array}$ & $1 / 1(313)$ & $\begin{array}{l}2 / 1-2(309, \\
313)\end{array}$ & $\begin{array}{l}2 / 2(308, \\
312)\end{array}$ & $1 / 1(315)$ & $\begin{array}{l}\text { P. europaea } \\
\text { (385) }\end{array}$ \\
\hline PA23 & $\begin{array}{l}(\mathrm{GAA})_{7} \mathrm{GGA} \\
(\mathrm{GAA})_{3}\end{array}$ & DQ665906 & $\begin{array}{l}\text { F: GGAGATAGCCACGAGACACC } \\
\text { R: CAAGCATCGCTGTAAACGAC }\end{array}$ & 155 & $62^{\circ} \mathrm{C}$ & $2 / 2+(133,148)$ & $\begin{array}{l}2 / 2(133 \\
148)\end{array}$ & $1 / 1(148)$ & $1 / 1(148)$ & $1 / 1(148)$ & $1 / 1(148)$ & - \\
\hline PA30 & $(\mathrm{AC})_{8}$ & DQ665907 & $\begin{array}{l}\text { F: TAGGGGACTTAAACCCCA } \\
\text { R: GTTGGCGGACTAGAGATTTT }\end{array}$ & 127 & $66^{\circ} \mathrm{C}$ & $\begin{array}{l}4 / 4+(120 \\
122,123,124)\end{array}$ & $\begin{array}{l}3 / 3+(120 \\
122,124)\end{array}$ & $1 / 1(123)$ & $2 / 2(118,122)$ & $\begin{array}{l}2 / 2(120 \\
122)\end{array}$ & $\begin{array}{l}2 / 2 \\
(120,122)\end{array}$ & - \\
\hline PA31 & $(\mathrm{CAA})_{8}$ & DQ665908 & $\begin{array}{l}\text { F: GCTTCTCACTGCACAGCAAC } \\
\text { R: AGGGTATTGGAGCCTGATGC }\end{array}$ & 195 & $62{ }^{\circ} \mathrm{C}$ & $\begin{array}{l}2 / 1-2+ \\
(183,192)\end{array}$ & 1/1 (192) & $\begin{array}{l}2 / 2(183, \\
192)\end{array}$ & $\begin{array}{l}4 / 3-4(174 \\
180,186,192)\end{array}$ & $1 / 1(150)$ & $1 / 1(150)$ & - \\
\hline
\end{tabular}

*, initial annealing temperature for touchdown PCR; + , loci with alleles consistently showing different peak areas. 
hypothesis that Pau and Pam may actually be the progenitors of Paa. Due to the uncertain nature of ploidy in these different taxa, expected heterozygosities and linkage disequilibria were not computed. However, depending on the locus, up to three or five different alleles were simultaneously observed in individual Pam and Paa isolates, respectively. In addition, genotyping of the PCR products obtained with the primer pairs PA3, PA6, PA8, PA11, PA14, PA23, PA30 and PA31 showed that for individual Paa and Pam isolates, the respective quantities of the different alleles (based on peak areas) were not always equal. The occurrence of unbalanced peak ratios suggests these species are polyploid (Christiansen 2006). The genotype amplification patterns for these loci will be particularly useful to unravel the genotypes of Paa and Pam.

\section{References}

Brasier CM, Cooke DEL, Duncan JM (1999) Origin of a new Phytophthora pathogen through interspecific hybridization. Proceedings of the National Academy of Sciences, USA, 96, 5878-5883.

Brasier CM, Kirk SA, Delcan J et al. (2004) Phytophthora alni sp. nov.
\& its variants: designation of emerging heteroploid hybrid pathogens spreading on Alnus trees. Mycological Research, 108, 1172-1184.

Christiansen DG (2006) A microsatellite-based method for genotyping diploid and triploid water frog of the Rana esculenta hybrid complex. Molecular Ecology Notes, 5, 190-193.

Dutech C, Amsellem L, Billotte N, Jarne P (2000) Characterization of (GA) microsatellite loci using an enrichment protocol in the neotropical tree species Vouacapoua americana. Molecular Ecology, 9, 1433-1435.

Estoup A, Solignac M, Harry M, Cornuet JM (1993) Characterization of $(\mathrm{GT})_{n}$ and $(\mathrm{CT})_{n}$ microsatellites in two insect species: Apis mellifera and Bombus terrestris. Nucleic Acids Research, 21, 14271431.

Ioos R, Andrieux A, Marçais B, Frey P (2006) Genetic characterization of the natural hybrid species Phytophthora alni as inferred from nuclear and mitochondrial DNA analyses. Fungal Genetics and Biology, 43, 511-529.

Rozen S, Skaletski H (2000) PRIMER3 on the WWW for general users and for biologist programmers. In: Bioinformatics Methods and Protocols: Methods in Molecular Biology (eds Krawetz S, Misener S), pp. 365-386. Humana Press, Totowa, New Jersey.

Weising K, Nybom H, Wolff K, Meyer W (1995) DNA Fingerprinting in Plants and Fungi. CRC press, Boca Raton, Florida. 